\section{Cahiers de Narratologie}

Analyse et théorie narratives

15 | 2008

Récits et genres historiques

\title{
Récit filmique et Histoire : La hiérarchie des personnages dans Goya en Burdeos de Carlos Saura (1999)
}

\section{Marc Marti}

\section{OpenEdition}

Journals

Édition électronique

URL : http://journals.openedition.org/narratologie/677

DOI : $10.4000 /$ narratologie. 677

ISSN : 1765-307X

Éditeur

LIRCES

Référence électronique

Marc Marti, «Récit filmique et Histoire : La hiérarchie des personnages dans Goya en Burdeos de Carlos Saura (1999) », Cahiers de Narratologie [En ligne], 15 | 2008, mis en ligne le 05 janvier 2011, consulté le 02 mai 2019. URL : http://journals.openedition.org/narratologie/677 ; DOI : 10.4000/ narratologie. 677

Ce document a été généré automatiquement le 2 mai 2019.

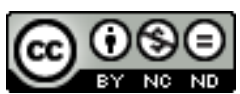

Cahiers de Narratologie - Analyse et théorie narratives est mis à disposition selon les termes de la licence Creative Commons Attribution - Pas d'Utilisation Commerciale - Pas de Modification 4.0 International. 


\title{
Récit filmique et Histoire : La
} hiérarchie des personnages dans Goya en Burdeos de Carlos Saura (1999)

\author{
Marc Marti
}

1 Goya en Burdeos de Carlos Saura est sans nul doute une œuvre qui ne peut être classée dans un genre précis. Dans cette étude, qui s'inscrit dans le cadre d'une réflexion sur les rapports entre le Récit et l'Histoire, il nous a cependant semblé intéressant d'envisager ce film sous l'angle de son rapport au genre historique, en particulier à propos de la construction et de la hiérarchisation des personnages.

D'abord, il sera indispensable de déterminer les rapports entre le genre historique et l'œuvre de Saura et de s'interroger ainsi sur sa nature même. Nous pourrons ensuite nous intéresser à la hiérarchisation des personnages pour analyser les rapports entre le système des personnages et l'Histoire et les problèmes spécifiques de la narration filmique sur ce point.

\section{Goya en Burdeos, film historique?}

\subsection{La problématique du genre}

3 Au premier abord, les relations que Goya en Burdeos entretient avec le genre historique ne semblent pas évidentes. Le film est par exemple répertorié comme «drame » dans la base de données du cinéma espagnol ${ }^{1}$. Cependant, le titre renvoie explicitement au personnage historique de Goya et aux cinq dernières années de sa vie : son exil volontaire et sa mort à Bordeaux (1823-1828). D'ailleurs, dans ce contexte, le Bordeaux de Saura est pratiquement vidé de sa substance spatiale. Le décor est en grande partie composé d'intérieurs dont l'exactitude référentielle n'est pas la principale caractéristique, comme le rappelle Carlos Saura lui-même dans une entrevue accordée à Jean-Claude Seguin ${ }^{2}$. Les 
rares extérieurs ne sont pas identifiables dans le sens où ils ne renvoient pas à un référent spatial pris dans le Bordeaux historique :

«Esa calle no es de Burdeos, es inventada. Está hecha también con panós

fotográficos $»^{3}$.

4 Le rôle de Bordeaux est finalement celui d'un référent temporel et non d'un référent spatial : c'est la période de l'exil pour Goya et pour l'Espagne libérale évoquée par le film, comme lors du dialogue entre le peintre et Moratín au début du film (séquence 4) ou plus loin lors de la réunion des libéraux chez Braulio Poc (séquence 18). De Bordeaux, il ne reste que quelques allusions (un rien stéréotypées) sur l'excellence de ses vins.

Globalement donc, le référent semble être l'Histoire, mais l'originalité réside dans son traitement. Si on admet ce préalable, parmi les nombreux sous-genres du cinéma historique, la biographie historique (biopic) est celui qui se rapproche du film de Saura tel que le présente le titre ${ }^{4}$. Cependant l'œuvre, selon les propres termes du réalisateur, est " un essai personnel sur un personnage $»^{5}$.

On remarquera d'abord que le propos n'est pas celui d'une œuvre didactique ou documentaire qui prétendrait à la reconstitution historique. Comme le souligne Pascale Thibaudeau, "nous sommes face à une œuvre de fiction qui utilise l'Histoire comme matériau à façonner ${ }^{6} »$. Il s'agit bien d'une Histoire subordonnée au récit filmique et non l'inverse, comme c'est souvent le cas dans les fictions dites «historiques ». En effet, ce genre a souvent été prisonnier à la fois de l'exigence "d'authenticité » et d'exactitude, propre à l'historiographie et des exigences du « réalisme » cinématographique ${ }^{7}$. Saura est clairement en rupture avec cette conception du cinéma historique et l'ensemble du film est soumis à la vraisemblance, catégorie artistique (et narrative dans le cas présent) et non à l'exactitude (au vrai), catégorie historiographique.

\subsection{Exactitude ou vraisemblance?}

7 Cependant, l'autonomie de l'œuvre par rapport à l'Histoire ne peut être complète et il convient de s'interroger sur les contraintes que distille le choix d'un personnage historique, malgré les libertés qui peuvent être prises avec l'exactitude.

Dans le récit de fiction historique, le public possède des références préalables, le patronyme Goya crée un horizon d'attente. Le personnage a existé et le public en possède sa propre représentation. On pourrait supposer que cette représentation repose sur plusieurs fondements: les portraits et autoportraits, les films antérieurs, l'institution scolaire et les biographies.

9 Les portraits et autoportraits sont les références de la ressemblance " physique » entre le personnage filmique et le personnage historique. Sur ce point, le réalisateur lui-même et les analyses universitaires signalent le troublant ajustement qui s'est opéré entre l'acteur Paco Rabal, le rôle et le personnage historique ${ }^{8}$.

Dans les films antérieurs sur le peintre, étudiés en partie par Jean-Claude Seguin, Goya joue principalement le rôle de "décorateur" et de référent historique non par son personnage mais par ses toiles, qui servent de fonds dans les films de José Buchs ou de Benito Perojo. Il faut attendre 1958 pour une première biographie produite par les studios d'Hollywood (The Naked Maja) et 1971 pour la première biographie espagnole (Goya, historia de una soledad). Puis, un an avant celui de Saura, sort le film de Bigas Luna Volaverunt ${ }^{9}$. À ces références cinématographiques, il conviendrait d'ajouter la série Goya, 
réalisée pour la télévision en 1985 et qui évoque l'ensemble de la vie du peintre. Les documentaires, un thème que n'aborde pas Jean-Claude Seguin, ont été plus nombreux que les fictions. En effet, la base de données du cinéma espagnol en recense dix d'origine nationale, dont les dates s'échelonnent entre 1944 à 1982: Aquel Madrid de Goya (1944), Goya (1948), Goya, una vida apasionada (1957), Goya, tiempo y recuerdo de una época (1960), La España de Goya (1970), Goya (1973), La mujer en Goya (1977), Los caprichos de don Francisco de Goya (1980), Un genio llamado Goya (1981), Sintesis interpretativa sobre varios lienzos de Goya (1982). Malgré ces précédents, Carlos Saura fait preuve d'originalité dans le choix du référent. Les dernières années de la vie du peintre ne sont traitées que dans la série télévisée. Cet élément montre que le film dépasse largement le genre historique, c'est aussi une réflexion sur la mort, la vie et l'art et il est évident que la puissance qui s'en dégage provient plus de la mise en dynamique des visions et des toiles du peintre que de la minutie dans le costume ou le détail.

11 En ce qui concerne les références littéraires et historiques, Saura choisit de les adapter à une trame narrative. En fonction des sujets, des époques et des lieux, le genre historique est susceptible d'osciller entre le documentaire et la fiction ${ }^{10}$. Les écarts par rapport à l'exactitude historique montrent clairement le choix esthétique qui a été fait. Le film privilégie comme éléments moteurs les amours de Goya et de la Duchesse d'Albe, une réalité dont doutent les biographes les plus scrupuleux, ce dont le réalisateur n'a cure ${ }^{11}$ :

«Que si han tenido una relación, que si la han tenido, que si no... Pero, ¿qué más

da ? Yo estoy de acuerdo con esta visión más o menos romántica ».

12 Ces amours tourmentés, au-delà de la mort dans l'œuvre de Saura, donnent la dynamique narrative indispensable au film. Cependant, ce choix esthétique au détriment d'éléments historiques " exacts » doit être relativisé. L'hypothèse des amours entre le peintre et Cayetana est une version très populaire de l'Histoire, sans nul doute la plus répandue parmi le public et la plus utilisée dans les fictions biographiques du peintre. On touche là un des paradoxes du récit de fiction historique en général. La fiction s'accommode mieux de références historiques "populaires", acceptées par le public (et porteuses d'une dynamique narrative) que de vérités historiques « savantes », parfois plus confidentielles.

Pour conclure provisoirement, on pourrait avancer que Goya en Burdeos est schématiquement au croisement de la biographie historique, du drame et de l'essai cinématographique. Le premier apporte les références et les personnages historiques, le second la trame narrative, fortement conditionnée par l'histoire d'amour, le troisième la vision personnelle du réalisateur sur l'art du peintre et l'art en général. Ces premières déterminations étant posées, on peut s'interroger sur la construction de la hiérarchie des personnages dans le film.

\section{Essai de hiérarchisation des personnages}

14 Pour le personnage de cinéma, à l'exception de l'ouvrage de Jean-Paul Aubert, les réflexions théoriques sont rares ${ }^{12}$. À notre connaissance, rien n'a été écrit sur le problème des personnages secondaires, pour lesquels il conviendrait d'envisager un début de définition. La liste des interprètes, qui fournit un ensemble fini et identifiable, peut servir de point de départ. Écartons rapidement les figurants, qui n'appartiennent pas vraiment aux personnages et qui ne « figurent pas » (justement) dans cette liste. Ils sont en grande partie, malgré l'anthropomorphisme de leur image, assimilables au décor. 
15 La première ligne de partage à tracer serait celle entre le (ou les) héros et les autres personnages. En l'absence d'une typologie cinématographique, nous pouvons recourir aux outils de l'analyse littéraire qui serviront de point de départ.

\subsection{Un essai de hiérarchisation : le héros et les personnages principaux}

Philippe Hamon, puis Vincent Jouve se sont posé la question du héros littéraire ${ }^{13}$. Ils rappellent en particulier que «le héros organise l'espace interne de l'œuvre en hiérarchisant la population de ses personnages $»^{14}$.

17 Dans le film, Goya doit être considéré comme le haut de la pyramide ou plutôt le point central du cercle des personnages. Autour de lui s'établit une hiérarchie essentielle dans l'organisation du récit. Pour les personnages principaux, on remarquera d'abord un léger décentrage, produit par le double rôle Goya vieux / Goya jeune, volontairement mis en relief par le choix de deux acteurs d'un âge différent (Paco Rabal et José Coronado). Cette double présence tend à créer deux personnages différents, le vieux apparaissant comme plus central que le jeune dans le sens où ce dernier est le produit d'une remémoration effectué par le premier. Il est en quelque sorte subordonné à son double. Cette duplication du héros fait partie du traitement du temps par le film. La linéarité, caractéristique fréquente du temps dans le récit historique, est brisée par de fréquentes analepses mais aussi par les coexistences d'époques et de temps différents dans la même scène, comme à la séquence, où Goya jeune est au chevet de Goya vieux (séquence 30).

Dans un premier cercle, on pourrait situer les trois figures féminines, toutes proches du peintre (Cayetana, Rosario, Leocadia), dans le sens où elles évoluent toutes dans son espace intime. Légèrement décentrée, la figure principale de l'ami, Moratín, qui partage au début du film un début d'intimité et réapparaît dans d'autres séquences $(4,11,18)$. C'est autour de ce groupe principal que se crée un système de personnages secondaires.

\subsection{Les personnages secondaires}

19 La notion de secondarité recouvre de fait un ensemble assez vaste, lui-même susceptible de distinctions internes. Le personnel secondaire du film est donc à son tour hiérarchisé.

Selon Philippe Hamon, l'analyse du héros littéraire se situe sur deux plans : d'une part le repérage de procédés stylistiques de différenciation, d'autre part, un système de valeurs, auquel renvoient ces mêmes procédés ${ }^{15}$. Les mécanismes différentiels, dits " de surface ", sont au nombre de six (qualification, distribution, autonomie, fonctionnalité, prédésignation, commentaire explicite). Ils n'opèrent pas tous obligatoirement, mais ils constituent les manifestations de surface de l'idéologie ou du système de valeurs qui soustend le texte. La méthode de Philippe Hamon, qui revendique sa filiation sémiologique, nous semble adaptable au cinéma. Cependant, pour qu'elle soit opératoire, il conviendra de prendre en compte les spécificités des différents ensembles sémiotiques qui le composent $^{16}$. Ces réflexions sur le héros sont aussi adaptées à l'analyse de l'ensemble des personnages. Elles devraient nous permettre d'arriver dans un premier temps à une hiérarchie avant de passer à une évaluation des personnages secondaires, leur(s) fonction (s) et leur signification(s) dans l'œuvre ainsi que leur rapport à l'Histoire. 

Coronado (Goya joven), Maribel Verdú (Duquesa de Alba), Eulalia Ramón (Leocadia), Dafne Fernández (Rosarito), Emilio Gutiérrez Caba (José de la Cruz), Joaquín Climent (Moratín), Manuel de Blas (Salcedo), Carlos Hipólito (Juan Valdés), Pedro Azorín (Braulio Poc), Joan Valles (Novales), Cristina Espinosa (Pepita Tudó), Paco Catalá (Asensio), Saturnino García (Cura / San Antonio), José María Pou (Godoy), Franco Di Francescantonio (Doctor en Andalucía), José Antonio (Bailarín Duques de Osuna), Mario De Candia (Bayeu), La Fura dels Baus (Los Desastres de la Guerra), Concha Leza (Mujer en Andalucía), Jaime Losada (Gaulon), Ainhoa Suárez (Rosarito 6 años), José Reche (Cadaver asesinado), José Sainz (Enterrador culpable), Demetrio Julián (Padre de San Antonio), Stephane Salom (Hombre joven francés), Roberto Arcilla (Hombre rollizo francés), Lorena Pellarini (Mujer francesa), Azucena de la Fuente (Josefina Bayeu), Bartolomé Moreno (Alguien 1), Francisco Jesús Santillana (Alguien 2), José Luis Chavarría (Alguien 3), Luis Llamas (Alguien 4), Nathalie Pinot (Profesora de piano), Olivier D'Belloch (Ayudante de Gaulon), Borja Elgea (Amigo de Goya).

Avant de s'intéresser au film, la liste des interprètes fournit déjà quelques éléments de hiérarchisation. On peut y distinguer trois groupes à partir des qualifications.

\subsection{L'ébauche d'une typologie}

D'abord, on distinguera les personnages à référent historique. Au nombre de 18 , ils constituent la moitié de la liste. Ils sont tous dotés d'un patronyme qui renvoie à une personne ayant existé.

Ensuite, on trouvera les personnages sans nom. Ils sont dotés soit d'une dénomination qui indique leur fonctionnalité (doctor en Andalucía, bailarín Duques de Osuna), soit désignés par un trait distinctif (mujer francesa, hombre rollizo francés), soit à peine identifiés (la série des 4 alguien).

Enfin, nous avons la catégorie, problématique dans sa définition comme nous le verrons, de ceux que nous appellerons les métapersonnages, qui interprètent deux créations de Goya (le miracle de Saint Antoine et les Désastres de la guerre).

Cette distinction des personnages à partir de la liste des interprètes doit cependant être mise à l'épreuve de l'analyse filmique qui devrait permettre de mettre en exergue leurs fonctions et leurs significations.

\section{Les personnages et l'Histoire : fonctions et significations}

\subsection{Les personnages marqués par l'histoire}

Si on s'en tenait à la liste des interprètes, on pourrait penser que le film s'inscrit pleinement dans le genre historique, en faisant la part belle aux personnages possédant un référent historique. En effet, ceux-ci forment la moitié du personnel de l'œuvre. Cependant, leur fonction référentielle dans le film est assez réduite. Une partie d'entre eux n'est pas nommée explicitement dans le film et c'est finalement le générique final qui en révèle le nom au moment où défile la liste des interprètes. C'est le cas des libéraux José de la Cruz, Novales, Juan Valdés, Asensio et de l'imprimeur Gaulón. Par ailleurs, Braulio 
Poc est désigné par l'enseigne à l'intérieur de la taverne, qui ne saute pas aux yeux du spectateur et c'est sa chanson qui permet de nommer un des libéraux (Salcedo). En dehors du cercle intime du peintre constitué par les sept personnages de Goya (2), Cayetana, Rosarito (2), Leocadia et Moratín, seuls sont nommés, alors qu'ils sont à l'écran, Godoy, Pepita Tudó et Josefina Bayeu. Francisco Bayeu est nommé mais lors d'une conversation entre Rosarito et le vieux Goya. La scène dans laquelle il apparaît ne donne aucune indication sur son identité. Ce dernier cas est même exemplaire d'un renoncement à la convention référentielle du genre historique. En effet, incarné par l'acteur italien Mario de Candia, Bayeu (qui était d'origine aragonaise) s'exprime en italien. On devine son identité uniquement parce qu'il rappelle à Goya qu'il attend la visite de sa sœur (femme de Goya) avec l'enfant.

Cette liberté prise avec l'exactitude historique perce aussi dans la scène du salon des Ducs d'Osuna. Pour le spectateur, la séquence n'offre aucune indication chronologique précise, mais Goya dit :

« Mi máxima ambición era ser pintor de Corte »

Cette réplique situerait la scène avant 1789, au moment où Goya fréquente les salons et essaie de faire avancer sa carrière comme le suggère le monologue intérieur. Si on en croit le scénario publié par Saura, cette séquence serait censée se passer en 1781, date à laquelle Goya a 35 ans $^{17}$. Saura lui fait rencontrer pour la première fois Cayetana au cours de cette même séquence. À ce propos, le peintre interroge ainsi son ami :

«-Quién es ? La que ríe, la que está con Godoy »

Cette réplique et les images qui suivent présentent un double anachronisme. D'abord, Godoy n'est arrivé à Madrid qu'en 1784, à l'âge de 17 ans, ensuite, il est incarné par un acteur plus âgé que José Coronado, alors que le futur Prince de la Paix avait vingt et un ans de moins que Goya. Le physique des personnages du film suggère pratiquement le contraire, José María Pou qui interprète Godoy est né en 1944 (55 ans au moment de la sortie du film) et José Coronado qui interprète Goya jeune est né en 1957 (42 ans en 1999). Cet extrait montre le rejet d'une convention propre au genre historique, celle de la reconstitution par l'exactitude. L'exemple est d'autant plus frappant que l'on se trouve dans une scène où le détail historique est particulièrement soigné : visages poudrés, perruques, mouches sur les joues selon la mode française du moment, costumes et mobilier d'époque (séquence 3).

31 La narration filmique de Goya en Burdeos est basée sur une remémoration qui « écrase » en quelque sorte le temps historique, un procédé que Saura a déjà utilisé par exemple dans La prima Angélica. Seuls Goya, Moratín, Leocadia et Rosarito semblent vieillir. Cayetana reste intemporelle et Godoy jeune est déjà Godoy vieux. Les anachronismes suggèrent que l'on se trouve dans les couloirs de la mémoire et non dans ceux du temps historique. Un exemple assez frappant se trouve après la scène où le peintre vient de visionner les «Majas » avec Godoy et quelques familiers du Prince de la Paix. Ici, Goya, (interprété encore par José Coronado), parcourt une galerie de portraits dans laquelle figure celui de Ferdinand VII en monarque, un tableau qu'il ne peindra que plusieurs années plus tard.

\subsection{Les personnages sans nom}

32 Ces personnages, malgré leur absence de patronyme, peuvent aussi renvoyer à l'Histoire. En effet, le médecin en Andalousie est une référence directe à la maladie du peintre 
contractée en 1792. Cependant, cette référence historique est largement transcendée par la vision onirique à laquelle elle donne lieu en fin de séquence 7 .

En ce qui concerne les autres personnages, il y a peu de choses à dire. Seule la professeur de piano est attestée dans la biographie du peintre. Le reste du personnel est fonctionnel. Il ne joue qu'un rôle de comparse et ne renvoie à aucun référent historique. Il sert simplement à donner la réplique aux personnages secondaires importants et aux personnages principaux.

On remarquera enfin que la fiction, en privilégiant la remémoration et la vision onirique comme procédé narratif, accorde un rôle aux métapersonnages et brouille aussi les niveaux de la fiction.

\subsection{Les métapersonnages}

On pourrait hésiter sur cette catégorie, car une grande partie de Goya en Burdeos repose sur une construction analeptique, fondée sur les souvenirs de Goya vieux, et sans doute une étude de la construction temporelle de l'œuvre serait des plus intéressantes. Dans cette perspective, la majorité des personnages possède un statut de personnage au second degré, puisqu'ils sont le produit des souvenirs du peintre. Les cas les plus flagrants sont constitués par Cayetana, qui semble intemporelle ou par Goya jeune qui est une remémoration de Goya vieux.

Cependant, cette analyse des personnages principaux comme métapersonnages nous éloignerait de notre sujet et nous associerons uniquement le concept de métapersonnages aux «peintures vivantes » que le film met en scène. Il s'agit ici de séquences qui se présentent de façon marquée comme des œuvres à l'intérieur de l'œuvre. Le film contient plusieurs tableaux vivants, mais seuls deux d'entre eux font appel véritablement à des métapersonnages, il s'agit du Miracle de Saint Antoine et Les désastres de la Guerre.

La fresque fait l'objet de deux séquences (12 et 14). Dans la première, on voit naître l'idée à partir d'une lecture -sans doute une vie de saint- faite tour à tour par Moratín, le prêtre et le peintre. La lecture débouche sur une vision, non dénuée d'ironie si on considère sa théâtralité, qui met en scène le miracle et où interviennent les personnages secondaires. La particularité de ce premier tableau, c'est la présence dans la vision du prêtre qui était venu rencontrer le peintre pour la commande dans la réalité et qui se retrouve sous les traits de Saint Antoine. Ce déplacement d'un élément du premier niveau $\mathrm{du}$ film et son inclusion dans un tableau au second niveau n'est pas isolé. En effet, la séquence suivante fondée sur le célèbre carton La pradera de San Isidro inclut à la fois l'artiste, qui se retrouve personnage dans sa propre œuvre et Cayetana.

Les désastres de la Guerre offrent une double originalité. D'abord, du point de vue de la relation entre les arts graphiques et le cinéma, il s'agit de l'insertion d'une série de gravures dans le film. Le procédé choisi par Saura est original car il efface ce qu'il conviendrait d'appeler « l'effet-citation » utilisé par exemple pour les Caprices : la matière graphique est retravaillée pour devenir cinématographique. Cependant, ce travail qui est déjà à l'œuvre dans la Pradera de San Isidro se singularise par la dynamique, le mouvement qu'impulse le déroulement chronologique. La temporalité qu'implique la représentation filmique rend compte de la dynamique narrative que possède la série de gravures, qui ne restent pas cantonnée à une somme statique comme c'est le cas pour les Caprices et dans une moindre mesure pour La pradera de San Isidro. 

comme tel. La série de gravures est interprétée par la troupe la Fura del Baus. Le personnage secondaire est ici un personnage collectif. Ce personnage renvoie à une masse anonyme dont la constitution est complexe : c'est un épisode de l'histoire d'Espagne et une œuvre de Goya. La matière historique est à nouveau proposée au spectateur dans une grande complexité. C'est tout à la fois l'histoire d'Espagne, sa vision par Goya et sa représentation par Saura.

\section{Conclusion}

Pour conclure, le film Goya en Burdeos se distingue du genre historique malgré son utilisation de l'Histoire et de personnages historiques. Le traitement des personnages montre qu'il s'agit d'une création artistique très personnelle qui s'éloigne de la norme «naturaliste» selon les propres termes du réalisateur. Ce que le film propose aux spectateurs, c'est aussi une rupture avec le didactisme du genre historique, à la fois dans le contenu et dans les formes.

41 Ce qui prime dans le contenu, c'est la narrativité de la vie du peintre, les éléments qui en font un récit et non ceux qui en feraient une description fidèle, exacte mais statique. On retrouve la séparation entre la véracité et la vraisemblance, renvoyant à l'opposition entre récit historiographique et récit fictionnel.

Du point de vue de la forme, la rupture est sans nul doute encore plus grande. Les procédés détaillistes et la narration linéaire sont battus en brèche au profit d'un traitement qui redistribue le temps, marquant clairement l'autonomie du cinéma dans son rapport à la matière historique. Ce que propose Saura, c'est un cinéma libéré de la forme narrative historique, un cinéma de la subjectivité et de l'introspection, de l'œil et de la mémoire.

\section{NOTES}

1. Consultée le 27 janvier 2007.

http://www.mcu.es/bbddpeliculas/cargarFiltro.do?layout=bbddpeliculas\&cache=init

2. De Goya a Saura, échos et résonnances, sous la direction de Jean-Paul Aubert et de Jean-Claude Seguin, Lyon, GRIMH-LCE-GRIMIA, 2005, Jean-Claude Seguin, « Carlos Saura, entrevista », p. 254.

3. Ibid, p. 254.

4. Dans l'ouvrage La representación cinematográfica de la historia, les auteurs proposent une typologie des films susceptibles d'appartenir au cinéma historique. Voir Monterde, José Enrique, Selva, Marta; Solà, Ana, La representación cinematográfica de la historia, Akal, Madrid, 2001, p. 141.

5. Pascale Thibaudeau, « Goya peintre et danseur », in De Goya a Saura..., p. 249.

6. Pascale Thibaudeau, "Goya peintre et danseur », in De Goya à Saura, sous la direction de JeanClaude Seguin et Jean-Paul Aubert, Le Grimh, 2005, p. 227.

Cahiers de Narratologie, 15 | 2008 
7. À ce propos, voir l'article "Surimpressions ", de Christian Marc BOSSENO, in Le paradoxe du personnage, Les rencontres du centre des écritures cinématographiques, Moulin d'Andé - CECI / Images en manœuvre Éditions, 2004, p. 80.

8. Voir Jean-Paul Aubert, "Goya en Burdeos, un portrait imaginaire ", in De Goya a Saura..., p. $184-185$.

9. Nous laissons de côté (sans aucune pudeur) le film X de Joe d'Amato mentionné dans l'article de Jean-Claude Seguin.

10. Voir l'article «Surimpressions », de Christian Marc BOSSENO, art cit., p. 80.

11. Jean-Claude Seguin, « Entrevista... », p. 249.

12. Jean-Paul Aubert, Pour une esthétique du personnage filmique, Paris, L'Harmattan, 2001.

13. Nous ne discuterons pas sur la terminologie, un point examiné par Vincent Jouve et nous considérerons que héros est synonyme de personnage principal. Philippe Hamon, «Pour un statut sémiologique du personnage » Poétique du récit, Paris, Seuil, Coll. Points, 1977, pp. 115-180 ; Texte et idéologie, Paris, PUF, 1984 ; Vincent Jouve, "Le héros et ses masques", Cahiers de narratologie, $\mathrm{n}^{\circ} 6$, Le personnage romanesque, Nice, CNA, 1995.

14. Philippe Hamon, résumé par Vincent Jouve, art. cit., p. 250.

15. Dans le cadre de ce travail nous ne discuterons pas des termes employés par le critique français car ce n'est pas notre propos initial. On pourrait cependant avancer que le travail sur le héros reste à compléter. À ce propos voir aussi l'article de Vincent Jouve, cité précédemment.

16. Les acteurs sont un élément spécifique au cinéma, surtout dans le cadre d'une hiérarchie des personnages. La plupart du temps, les rôles secondaires sont tenus par des acteurs "moins connus " que ceux qui jouent les rôles principaux. C'est le cas dans Goya en Burdeos où le couple Francisco Rabal / Maribel Verdú possède une notoriété supérieure à celle des autres interprètes.

17. Voir Jean-Paul Aubert, «Goya en Burdeos, découpage séquentiel et synopsis », in " De Goya a Saura... ", p. 266, note 4 .

\section{RÉSUMÉS}

Goya en Burdeos de Carlos Saura est sans nul doute une œuvre qui ne peut être classée dans un genre précis. Dans cette étude, qui s'inscrit dans le cadre d'une réflexion sur les rapports entre le Récit et l'Histoire, il nous a cependant semblé intéressant d'envisager ce film sous l'angle de son rapport au genre historique, en particulier à propos de la construction et de la hiérarchisation des personnages.

Goya en Burdeos, es una obra de difícil clasificación genérica. En el estudio que sigue, nos pareció interesante, en el marco de las relaciones entre el Relato y la Historia, considerar la película bajo el aspecto de su relación con el cine histórico, en particular respecto a la construcción y la jerarquía de los personajes.

\section{INDEX}

Index chronologique : XXe siècle Index géographique : Espagne

Mots-clés : Carlos Saura, Goya, personnage filmique, sémiologie, récit, genre historique 
AUTEUR

MARC MARTI

Université de Nice-Sophia Antipolis. CIRCLES EA 3159 